\title{
Nontoxigenic Corynebacterium diphtheriae Isolated from Intravenous Drug Users
}

\author{
Eva Gruner, Milos Opravil, Martin Altwegg, \\ and Alexander von Graevenitz
}

\author{
From the Institute of Medical Microbiology. University of Zurich; and the \\ Division of Infectious Diseases. Department of Medicine. University \\ Hospital, Zurich. Switzerland
}

\begin{abstract}
During a prospective study 117 intravenous drug users were screened for infection with Corynebacterium diphtheriae. Nontoxigenic $C$. diphtheriae was found in 5 of 132 throat swab specimens and in 5 of 28 skin ulcer specimens taken from July 1991 to April 1992. When phenotypic and molecular typing methods were used, these 10 strains were shown to belong to a single clone. During the same period no strain was isolated from 200 controls. Clinical manifestations of infection were not clearly attributable to $C$. diphtheriae - no typical membranous pharyngitis was present. The presence of a single clone among homeless intravenous drug users in Zurich indicates the presence of $C$. diphtheriae in parts of the population with poor standards of hygiene and low socioeconomic status.
\end{abstract}

Diphtheria is expected to be eliminated in immunized populations of industrialized countries, with toxigenic strains no longer circulating among indigenous inhabitants [1]. Nevertheless, sporadic cases of diphtheria continue to be reported. with outbreaks probably limited to the lower socioeconomic levels of these populations [2-4]. The site of infection is often the skin rather than the pharynx, and toxic manifestations are unusual. Host resistance factors seem to influence susceptibility to infection with toxigenic as well as with nontoxigenic Corynebacterium diphtheriae [1,2].

Recently we reported three cases of endocarditis and one case of septicemia that occurred among intravenous drug users during the two winter seasons of 1990 and 1991 in Zurich [5]. Molecular typing of these four nontoxigenic isolates showed that they belonged to a single clone [6]. In consequence we wanted to know whether there was a reservoir of $C$. diphtheriae among the intravenous drug addicts living in Zurich. We present the results of our prospective study.

From July 1991 to April 1992, 117 intravenous drug users cared for at the infirmary for the homeless of the City of Zurich Medical Service and at the outpatient clinic of the Division of Infectious Diseases, Department of Medicine, University Hospital, Zurich, entered the study. Every person was examined with use of a pharyngeal swab and, in the presence of ulcerating skin lesions, an additional wound swab. If multiple pharyngeal swab specimens were collected, the second swab was included in the study only if it was obtained after an interval of $\geqslant 2$ months. A total of $160 \mathrm{swab}$ specimens (pharyngeal, 132; superficial wounds, 28) were

Received 9 April 1993; revised 21 June 1993.

Reprints or correspondence: Dr. Milos Opravil, Division of Infectious Diseases, Department of Medicine, University Hospital, Rämistrasse 100 , CH-8091 Zurich, Switzerland.

Clinical Infectious Diseases 1994;18:94-6

(C) 1994 by The University of Chicago. All rights reserved. 1058-4838/94/180!-0010\$02.00 collected. The individuals (males, $68 \%$; females, $32 \%$ ) were 17-45 years of age. Approximately one-half of the patients were infected with the human immunodeficiency virus (HIV). Their immunization status with respect to diphtheria was not known.

Two hundred pharyngeal swabs were taken consecutively during the same period from patients with pharyngitis who were included as controls. These individuals had consulted their physicians about pharyngitis; belonged to the same age group (range, 18-40 years); and lived in the same area but were not drug users.

All swabs were plated onto Columbia agar base (BectonDickinson, Cockeysville, MD) with 5\% sheep blood and onto cystine-tellurite blood agar (CTBA) [7] and were incubated aerobically at $37^{\circ} \mathrm{C}$ for 24 hours. Colonies suspected of harboring $C$. diphtheriae were identified according to the methods of Saragea et al. [8]. Toxigenicity was tested in guinea pigs and with use of the polymerase chain reaction [9]. Further enzyme activities and carbohydrate fermentation reactions were assayed using the API ZYM and API Coryne systems (API BioMérieux, La Balme, France). Susceptibility to the eight antibiotics commonly used to treat gram-positive infections (penicillin, erythromycin, netilmicin, streptomycin, tetracycline, chloramphenicol, rifampin, and vancomycin) was tested. For molecular typing genomic DNA was isolated, digested with three restriction endonucleases (EcoRI, HindIII, or $P v u I I$ ), and hybridized with a biotin-labeled pBR322 derivative containing a rDNA operon of Escherichia coli. All these typing methods have been described before [6].

Five (4.3\%) of 117 primary pharyngeal swab specimens and $5(17.9 \%)$ of 28 swab specimens from superficial wounds were positive for $C$. diphtheriae (table 1). None of the patients had $C$. diphtheriae isolated in cultures of both throat and wound specimens. Two patients with positive pharyngeal swab specimens suffered from tonsillitis without developing the typical membranes and were successfully treated with erythromycin and flucloxacillin, respectively. One pa- 
Table 1. Characteristics of patients with $C$. diphtheriae infection.

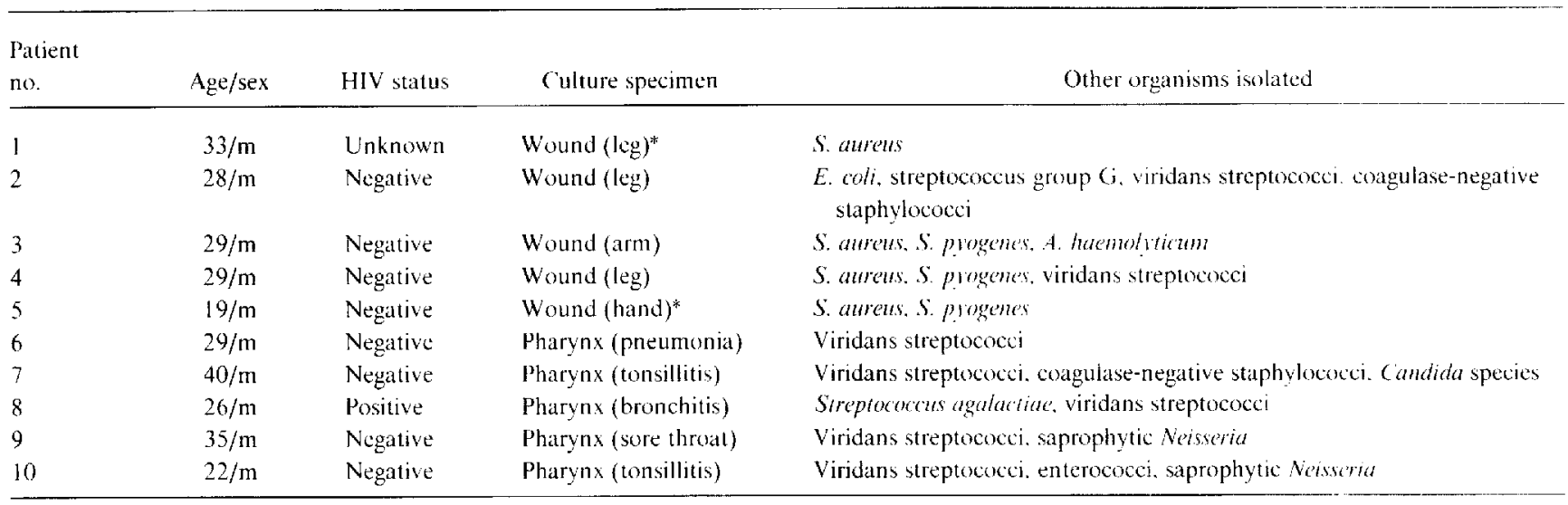

* Wound swab positive again after 2 and 5 months, respectively

tient suffered from a common cold with concomitant sore throat, and the remaining two had bronchopulmonary infections without pharyngeal symptoms. Both pharyngeal carriers who were treated and reexamined were found to be culture negative 10 and 15 days later, respectively. The five patients with positive wound swab specimens had skin infections and ulcers at the sites of drug injection. Figure 1 illustrates multiple ulcers on the calf of a patient, cultures of which yielded C. diphtheriae and Siaphylococcus aureus. This patient was treated with amoxicillin/clavulanate for 2 weeks, followed by erythromycin for 1 week. resulting in clinical improvement: however, he relapsed 2 months later with infection due to the same flora. The other patients were treated with amoxicillin/clavulanate with variable success as a result of poor compliance and persistent drug abuse. Only one of them presented again after 5 months, at which time diphtheric bacilli were still detected in swab specimens. HIV serology was available for nine of the culture-positive patients: only one pharyngeal carricr was HIV-positive.

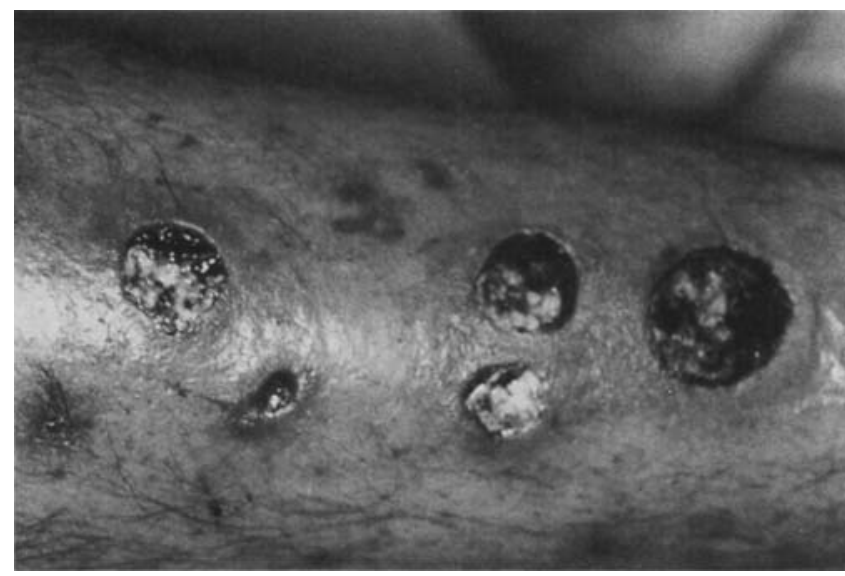

Figure 1. Calf of an intravenous drug user with multiple skin ulcers ( $1-3 \mathrm{~cm}$ in diameter); culture of the wound swab yielded $C$. diphtheriae and $S$. aureus.
In pharyngeal swabs $C$. diphtheriac was found together with the normal flora of the oropharynx, whereas in skin lesions the organism was associated with $\beta$-hemolytic streptococci (four of five). S. aureus (four). Arcanobacterium haemolyticum (one). E. coli (onc), and viridans streptococci (two) (table 1). This corresponds to data reported earlicr. in which the majority ( 73 ) of diphtherial skin lesions was associated with Streptococcus progenes infection [2]. However, in our patients coinfection by $S$. aureus was less frequent $(35 \%)$ [2]. No C. diphtheriae strains were isolated from the pharyngeal swabs of the 200 control patients who were not intravenous drug users.

The 10 isolates of $C$ diphtheriae were identified as biotype mitis by virtue of their inability to ferment dextrin. starch. and glycogen [8]. They were all identical with respect to their biochemistry. and they were all resistant to tetracycline but susceptible to all other antibiotics tested.

The genome of biotype mitis strains has been described as particularly variable in contrast to biotype intermedius [10], and this variability was confirmed in our former study by comparison with rDNA patterns of eight other mitis strains isolated in Switzerland over the past 5 years [6]. However. with each of the three restriction enzymes, the present isolates showed rDNA patterns that were identical to those observed previously in the isolates from intravenous drug users with endocarditis and septicemia [6]. We believe. therefore. that they all belong to the same clone.

Although $C$. diphtherike is only a human parasite, its isolation has become uncommon in microbiological laboratories of the western world. Its colonial morphology does not differ from that of many other gram-positive rods. and selective media such as CTBA have to be used if there is clinical suspicion of diphtheria. Otherwise, toxigenic as well as nontoxigenic strains are simply reported as coryneform bacteria on the assumption that they belong to the normal flora of the skin or the upper respiratory tract. Thus it is difficult to estimate the prevalence of $C$. diphtheriae in immunized popu- 
lations. During the early 1980 s Naumann et al. [3] found $C$. diphtheriae in $<0.1 \%$ of a German population [3]. It has recently been reported that screening of $>500$ healthy adults in an urban area of central Italy did not yield any pharyngeal carriers of either toxigenic or nontoxigenic diphtheric bacilli [11]. Similarly, none of the patients from our control group carried $C$. diphtheriae in his throat.

Our data reflect the continued decline in the incidence of diphtheria in areas where vaccination has been extensively practiced [1]. However, the reason for the reduction in prevalence of the bacterium itself is unclear, as vaccination is directed against the action of the toxin, and several studies in industrialized countries have demonstrated nonprotective levels of antitoxin titers in both children and adults despite vaccination $[1,12]$. The decline in diphtheria cases might, therefore, parallel development of the socioeconomic status, rather than the vaccination status, of the population. In fact, diphtheria or atypical manifestations of disease caused by nontoxigenic strains continue to be reported $[2,4]$ but are mostly limited to persons living under poor socioeconomic conditions. Outbreaks among alcoholics and homeless people have been described in Seattle [2, 10] and in Stockholm [4], where pharyngeal or generalized diphtheria occurred in people with insufficient levels of immunity.

Skin infections were described as chronic, nonhealing ulcers due to physical trauma and/or underlying dermatoses that were probably superinfected with predominantly nontoxigenic strains of $C$. diphtheriae. Thus, poor hygiene and personal proximity seem to favor colonization; these factors may also be responsible for the often-cited higher contagiousness of skin vs. pharyngeal diphtheria [13]. Our patients in whom $C$. diphtheriae was detected were homeless, except for one pharyngeal carrier who lived in his own apartment. However, they all slept in different homeless shelters and no close contacts between them could be identified. The transmission might have occurred among the intravenous drug users who congregated in one park (Platzspitz) of the city at that time. It is surprising that the HIV status of the patients did not seem to influence either the rate or the severity (e.g., endocarditis or sepsis) of infection with C. diphtheriae in this setting.

This study illustrates the persistence of $C$. diphtheriae in parts of the population. The presence of a single clone among intravenous drug users in Zurich indicates that specific conditions have to be met to maintain colonization with C. diphtheriae. These conditions include poor standards of hygiene and low socioeconomic status. Overcrowding in households and personal proximity seem to favor dissemination of cutaneous flora. Despite extensive immunization programs in developed countries, persons of low socioeconomic status continue to be at risk for colonization and infection with diphtheric bacilli.

\section{Acknowledgment}

The authors express their gratitude to Dr. A. Studer, City of Zurich Medical Service, for the care of the patients.

\section{References}

1. Karzon DT, Edwards KM. Diphtheria outbreaks in immunized populations. N Engl J Med 1988:318:41-3.

2. Harnisch JP, Tronca E. Nolan CM. Turck M. Holmes KK. Diphtheria among alcoholic urban adults. A decade of experience in Seattle. Ann Intern Med 1989;111:71-82.

3. Naumann P, Krech T, Maximescu P, et al. Phagenlysotopie und die Epidemiologie der Diphtherie-Erkrankungen 1975 bis 1984. Dtsch Med Wochenschr 1986:111:288-92.

4. Rappuoli R. Perugini M, Falsen E. Molecular epidemiology of the 1984-1986 outbreak of diphtheria in Sweden. N Engl J Med 1988;318:12-4.

5. Zuber PLF, Gruner E, Altwegg M, von Graevenitz A. Invasive infection with non-toxigenic Corynebacterium diphtheriae among drug users. Lancet 1992:339:1359.

6. Gruner E, Zuber PLF. Martinetti Lucchini G, von Graevenitz A, Altwegg M. A cluster of non-toxigenic Corynebacterium diphtheriac infections among Swiss intravenous drug abusers. Med Microbiol Lett 1992:1:160-7.

7. Frobisher M Jr. Cystine-tellurite agar for C. diphtheriae. J Infect Dis 1937;60:99-105.

8. Saragea A, Maximescu P, Meitert E. Corynebacterium diphtheriae: microbiological methods used in clinical and epidemiological investigations. In: Bergan T, Norris JR, eds. Methods in microbiology. Vol 13. New York: Academic Press. 1979:61-176.

9. Martinetti Lucchini M, Gruner E. Altwegg M. Rapid detection of diphtheria toxin by the polymerase chain reaction. Med Microbiol Lett 1992:1:276-83.

10. Coyle MB, Groman NB, Russell JQ, Harnisch JP, Rabin M, Holmes KK. The molecular epidemiology of three biotypes of Corynebacterium diphtherice in the Seattle outbreak, 1972-1982. J Infect Dis 1989;159:670-9.

11. Mencarelli M, Zanchi A, Cellesi C, Rossolini A, Rappuoli R, Rossolini GM. Molecular epidemiology of nasopharyngeal corynebacteria in healthy adults from an urban area where diphtheria vaccination has been extensively practiced, Eur J Epidemiol 1992;8:560-7.

12. Björkholm B. Böttiger M. Christenson B. Hagberg L. Antitoxin antibody levels and the outcome of illness during an outbreak of diphtheria among alcoholics. Scand J Infect Dis 1986;18:235-9.

13. Belsey MA. Isolation of Cormebacterium diphtheriae in the environment of skin carriers. Am J Epidemiol 1970;91:294-9. 